\title{
ESTRATIFICAÇÃO VERTICAL EM FLORESTA OMBRÓFILA DENSA DE TERRA FIRME NÃO EXPLORADA, AMAZÔNIA ORIENTAL ${ }^{1}$
}

\author{
Deoclides Ricardo de Souza² e Agostinho Lopes de Souza ${ }^{3}$
}

\begin{abstract}
RESUMO - Este estudo foi conduzido na Unidade de Manejo Florestal (UMF) da Fazenda Tracajás (02³5'53'S e 4747'10”W), no município de Paragominas, Estado do Pará, Brasil, e teve como objetivo a estratificação vertical da floresta ombrófila densa de terra firme não explorada, empregando-se análises de agrupamento e discriminante. A floresta foi estratificada em três áreas homogêneas, denominadas classes I, II e III de estoques volumétricos. Em cada classe de estoque volumétrico foram instaladas, aleatoriamente, cinco parcelas de 100 x $100 \mathrm{~m}$ (1,0 ha) cada uma, cujas alturas totais de árvores individuais com dap $\geq 15 \mathrm{~cm}$ foram utilizadas na estratificação vertical da floresta. As árvores individuais foram organizadas em ordem crescente de altura total e classificadas em classes de $1 \mathrm{~m}$ de amplitude, desde a altura total mínima até a altura total máxima. Elaborouse uma matriz $X$ de altura total, em que cada variável $\mathrm{x}_{\mathrm{ij}}$ representou a altura total da $i$-ésima árvore classificada na j-ésima classe de altura total. A matriz $X$ foi utilizada como input nas análises de agrupamento e discriminante. A aplicação da análise de agrupamento, utilizando o método de Ward, resultou em agrupamentos hierárquicos e seqüenciais das classes de altura em estratos de altura total (inferior, médio e superior). A análise discriminante, utilizando o método de Fisher, evidenciou que a classificação foi 100\% correta. A subdivisão da estrutura vertical da floresta em estratos de altura total com o emprego da análise multivariada mostrou-se um método eficiente e exeqüível de estratificação vertical de florestas ineqüiâneas.
\end{abstract}

Palavras-chave: Floresta tropical, estratificação vertical, análise multivariada.

\section{VERTICAL STRATIFICATION OF AN UNEXPLORED TERRA FIRME DENSE OMBROPHYLOUS FOREST, EASTERN AMAZON}

\begin{abstract}
ABSTRATCT - This study was carried out at the Forest Management Unit (FMU) in Tracajás farm (02³5'53"' $\mathrm{S}$ and $\left.47^{\circ} 47^{\prime} 10^{\prime \prime} \mathrm{W}\right)$, owned by Nova Era Agroflorestal, in Paragominas, Pará, Brazil. The objective was to accomplish the vertical stratification of an unexplored terra firme dense ombrophylous forest using cluster and discriminant analysis. The forest was stratified in homogeneous areas, denominated classes of volumetric stock I, II and III. In each class, five $100 \times 100 \mathrm{~m}$ plots were randomly set up, where the total heights of the individual trees $(d b h \geq 15.0 \mathrm{~cm})$ were used for vertical forest stratification. The trees were organized in increasing order of total height and classified in $1 \mathrm{~m}$ amplitude classes, from the minimum to the maximum total height. A total height $\mathrm{X}$-matrix was elaborated, with each variable $\mathrm{x}_{\mathrm{ij}}$ representing the total height of the $\mathrm{i}$-th tree classified into $\mathrm{j}$-th class of total height. This $\mathrm{X}$-matrix was the input for the cluster and discriminant analysis. The application of cluster analysis resulted into hierarchical and sequential clusters of height classes
\end{abstract}

\footnotetext{
${ }^{1}$ Recebido para publicação em 21.10.2003 e aceito para publicação em 10.8.2004.

${ }^{2}$ Departamento de Engenharia Agronômica da Universidade Federal de Sergipe - UFS. 49100-000 - São Cristovão, SE. <souzadr@hotmail.com>.

${ }^{3}$ Departamento de Engenharia Florestal da Universidade Federal de Viçosa. 36570-000, Viçosa, MG.<alsouza@mail.ufv.br>.
} 
into total height strata (inferior, medium, and superior). Discriminant analysis demonstrated a $100 \%$ correct classification. The vertical structure subdivision of forest into total height strata, by multivariate statistics proved the method efficient for uneven-aged forest vertical stratification.

Key words: Tropical forest, vertical stratification, multivariate analysis.

\section{INTRODUÇÃO}

$\mathrm{Na}$ análise estrutural de florestas tropicais ineqüiâneas, seja para fins de estudos fitossociológicos, seja para fins de manejo florestal, a estrutura vertical é um importante indicador de sustentabilidade do manejo florestal (SOUZA et al., 2003). Dependendo da região fitoecológica, do estádio de sucessão e do estado de conservação, a estrutura florestal pode apresentar, por exemplo, sub-bosque, estrato inferior, estrato médio e estrato superior, ou pode, na maioria das vezes, não se apresentar estratificada dessa forma.

As florestas heterogêneas ineqüiâneas bem estratificadas apresentam maior diversidade e diferenciação de nichos ecológicos e suportam maior diversidade de plantas e animais nos distintos estratos verticais, dentro de uma mesma comunidade vegetal (HUNTER Jr., 1990). Essa diferenciação pode ser determinada, sobretudo, pela resposta do decréscimo da disponibilidade de luz ao longo do perfil vertical, em direção ao piso da floresta.

O número de estratos é uma peculiaridade de cada floresta associada às diferenças em composição de espécies, relações competitivas, restrições ambientais e perturbações antrópicas ou naturais (LATHAM et al., 1998).

Embora haja vários métodos de estratificação de alturas de comunidades vegetais arbóreas (VEGA, 1966; LONGHI, 1980; LAMPRECHT, 1990; MARISCAL FLORES, 1993; CALEGÁRIO et al., 1994; SANQUETA, 1995), as técnicas estatísticas multivariadas, sobretudo as análises de agrupamento e discriminante, são as de maior potencial de aplicação em estudos da estrutura vertical (SOUZA et al., 2003). Essas técnicas são utilizadas com objetivos básicos de ordenamento e de agrupamento, com o propósito de classificação (BURTON et al., 1991).

Nesse sentido, este estudo teve como objetivo a estratificação vertical de uma floresta ombrófila densa de terra firme não explorada, empregando-se as técnicas de análises de agrupamento e discriminante.

\section{MATERIAL E MÉTODOS}

O estudo foi realizado na unidade de manejo florestal (UMF) da Fazenda Tracajás (02³5'53'S e 4747'10”'W), empresa Nova Era Agroflorestal, de propriedade do Grupo Rosa Madeireira, município de Paragominas, Estado do Pará, Brasil. A tipologia florestal é classificada como floresta ombrófila densa de terra firme em estádio de sucessão primária (clímax).

Na execução do plano de manejo florestal, destinado à produção de madeira para serraria e laminação, foi realizado o inventário de prospecção (100\%), no qual se estimaram os volumes do fuste comercial das árvores com dap $\geq 45 \mathrm{~cm}$ de 55 espécies comerciais em 49 talhões de exploração de 10 ha cada um, perfazendo um total de 490 ha.

No inventário $100 \%$ das árvores comerciais com $d a p \geq 45 \mathrm{~cm}$ (diâmetro mínimo de corte permissível) foram identificadas e marcadas as árvores-matriz, as árvores localizadas nas áreas de proteção ambiental e as árvores selecionadas para corte.

A unidade de manejo florestal, isto é, os 49 talhões de exploração, foi estratificada em áreas homogêneas de florestas, com base no estoque de árvores comerciais das 55 espécies autorizadas para corte pelo IBAMA, doravante denominadas classes I, II e III de estoque volumétrico. Foram empregadas as técnicas de análises de agrupamento (método de Ward) e discriminante (método de Fisher) na definição das referidas classes de estoque.

$\mathrm{Na}$ análise de agrupamentos, os talhões ou unidades de trabalho formaram grupos homogêneos e distintos, agrupados nas classes I, II e III de estoques volumétricos, com os respectivos volumes mínimo, médio, máximo e desvios-padrão (Quadro 1).

Em cada classe de estoque foram instaladas aleatoriamente cinco parcelas de $100 \times 100 \mathrm{~m}(1,0 \mathrm{ha})$ cada uma, no sentido leste-oeste, para medição dos indivíduos com dap $\geq 15 \mathrm{~cm}$. O diâmetro a $1,30 \mathrm{~m}$ do solo (dap) foi medido com uma fita métrica e a altura total dos indivíduos, com uma vara graduada em metro. 
Quadro 1 - Caracterização das classes de estoques volumétricos, mediante os valores dos volumes mínimo, médio e máximo, e dos desvios-padrão

Table 1 -Characterization of volumetric stock classes, value of standart deviation, volumes maximum, average and minimum

\begin{tabular}{lcccc}
\hline $\begin{array}{l}\text { Classe de } \\
\text { Estoque }\end{array}$ & Mínimo & \multicolumn{3}{c}{ Volume } \\
Médio & Máximo & Desvio-Padrão \\
\hline I & 35,60 & 45,16 & 50,89 & 4,67 \\
II & 52,55 & 58,11 & 63,82 & 3,41 \\
III & 69,50 & 76,77 & 89,36 & 6,37 \\
\hline
\end{tabular}

Cada parcela permanente foi demarcada com piquetes e identificada com plaqueta de madeira pintada de branco.

As alturas totais de árvores individuais com $d a p \geq 15 \mathrm{~cm}$ foram utilizadas na estratificação vertical.

No método de estratificação utilizado (SOUZA et al., 2003), empregaram-se as técnicas de análises de agrupamento (método de Ward) e discriminante (método de Fisher), com os seguintes procedimentos: ordenação crescente das alturas das árvores individuais; reunião das alturas totais em classes com amplitudes de $1 \mathrm{~m}$; e elaboração de uma matriz $X$ de dados de classes de altura total, em que a variável $\mathrm{x}_{\mathrm{ij}}$ representa a altura total da $i$-ésima árvore classificada na $j$-ésima classe de altura.

A matriz $X$ foi utilizada como input das análises de agrupamento e discriminante. Utilizaram-se a distância euclidiana simples e o método de Ward, pelo emprego das expressões:

$$
\begin{aligned}
d_{i j} & =\sqrt{\sum_{h=1}^{p}\left(x_{h i}-x_{h j}\right)^{2}, \mathrm{~h}=1,2, \ldots ., \mathrm{P}} \\
d_{i j}^{2} & =\left(x_{i}-x_{j}\right)^{2}
\end{aligned}
$$

em que $\mathrm{d}_{\mathrm{ij}}=$ distância euclidiana estimada entre as classes i e j, $x_{h i}=$ altura total da h-ésima árvore na i-ésima classe de altura, $\mathrm{x}_{\mathrm{hj}}=$ altura total da h-ésima árvore na j-ésima classe de altura total e $\mathrm{d}^{2}{ }_{\mathrm{ij}}=$ distância entre as médias dos grupos I e J.

A análise discriminante foi utilizada para verificar a distinção e a classificação dos estratos de altura total obtidos pela análise de agrupamento.
A composição florística foi analisada com base na riqueza ou no número de espécies (S), na diversidade máxima $\left(\mathrm{H}_{\text {máx }}\right)$ e no índice de diversidade de Shannon-Weaver (H') (BROWER e ZAR, 1984).

Os parâmetros fitossociológicos da estrutura horizontal, como a densidade absoluta e a dominância absoluta, foram estimados conforme Muller-Dombois e Ellemberg (1974).

O volume de fuste com casca de árvores individuais foi estimado pelo emprego da equação $\hat{Y}=0,0774759688+0,517896768\left(d_{a p}^{2} \times H c\right)(3)$, desenvolvida por Queiroz (1984).

\section{RESULTADOS E DISCUSSÃO}

Nos dendrogramas obtidos da análise de agrupamento (Figuras 1, 2 e 3), o eixo vertical apresenta a distância euclidiana simples em porcentagem e o eixo horizontal, as classes de altura total em metros, nas classes I, II e III de estoques volumétricos, respectivamente.

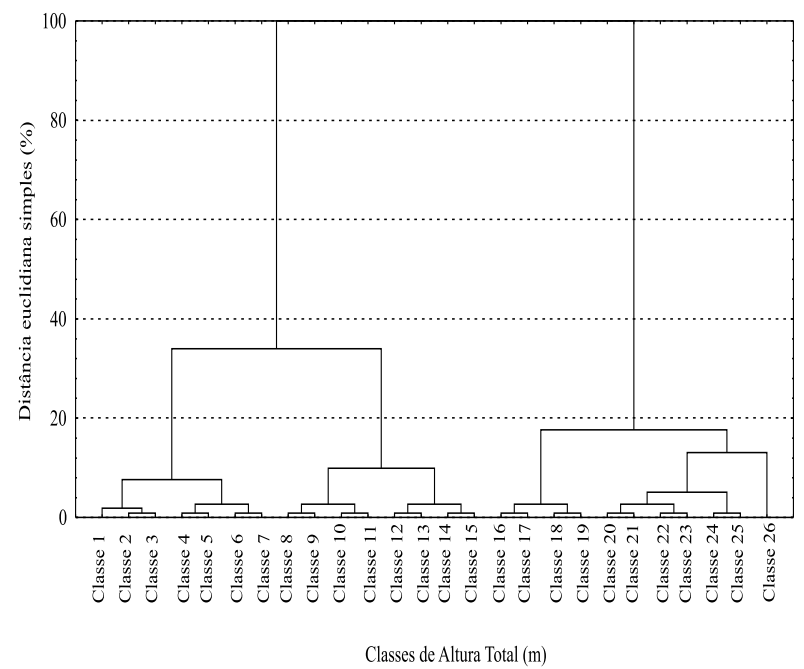

Figura 1 - Dendrograma mostrando o agrupamento das classes de altura total, classe I de estoque, obtido da análise de agrupamento utilizando a distância euclidiana simples e o método de Ward. Fazenda Tracajás, município de Paragominas, Estado do Pará.

Figura 1 -Dendrogram the cluster of total height classes, stock class I, obtained from cluster analysis using Eucliddean distance and Ward method. Tracajás Farm, municipality Paragominas, Pará state. 


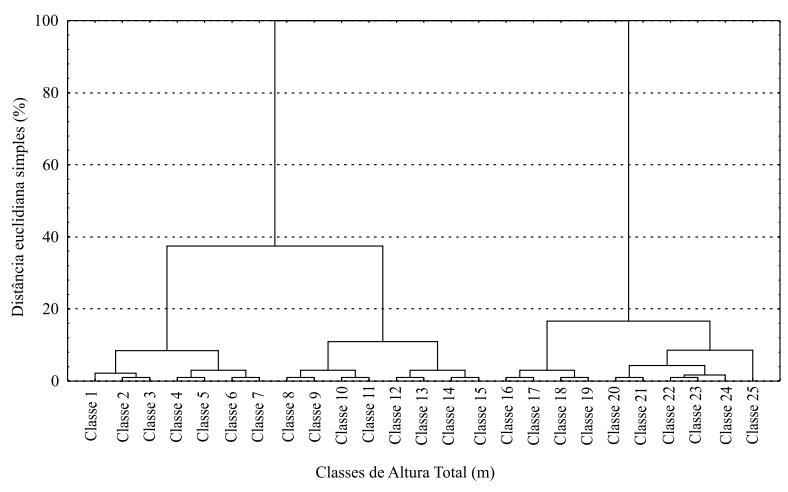

Figura 2 - Dendrograma mostrando o agrupamento das classes de altura total, classe II de estoque, obtido da análise de agrupamento utilizando a distância euclidiana e o método de Ward. Fazenda Tracajás, município de Paragominas, Estado do Pará.

Figura 2 -Dendrogram of cluster of total height class, stock class II, obtained from cluster analysis using Eucliddean distance and Ward method, Tracajás Farm, municipality Paragominas, Pará stat.

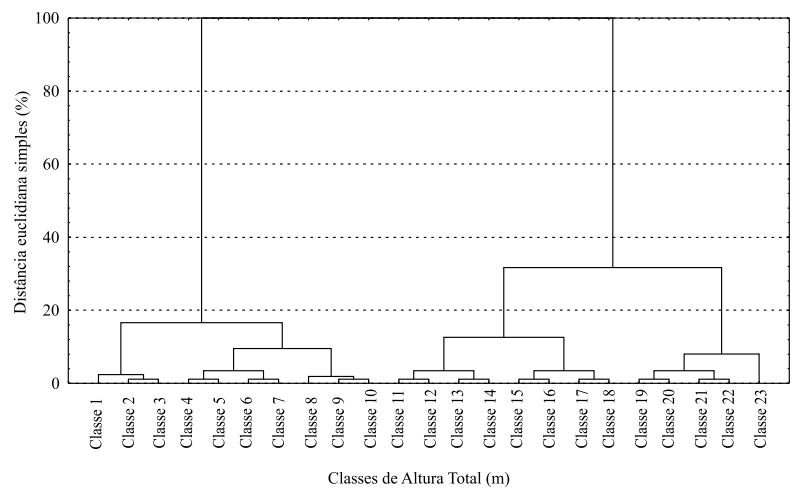

Figura 3 - Dendrograma mostrando o agrupamento das classes de altura total, classe III de estoque, obtido da análise de agrupamento utilizando a distância euclidiana e o método de Ward. Fazenda Tracajás, município de Paragominas, Estado do Pará.

Figura 3 -Dendrogram of cluster of total height class, stock class III, obtained from cluster analysis using Eucliddean distance and Ward method, Tracajás Farm, municipality Paragominas, Pará state.

$\mathrm{Na}$ análise dos dendrogramas (Figuras 1, 2 e 3), foram traçadas linhas de corte no nível de homogeneidade de $20 \%$, indicando três grupos homogêneos e distintos de classes de altura total, denominados estratos de altura total (I, II, III). O arranjo das árvores individuais em amplitudes de $1 \mathrm{~m}$ permitiu agrupar, seqüencialmente, em ordem crescente as classes de altura total, por meio do emprego da distância euclidiana simples e do método de Ward (Figuras 1, 2 e 3).

A análise de agrupamento executada na classe I de estoque (Figura 1) indicou que as classes de altura total de 1 a 7 formam o estrato inferior (I), as classes de altura total de 8 a 15 , o estrato médio (II) e as classes de altura total de 16 a 26 , o estrato superior (III).

A análise discriminante apresentou probabilidades de 0,$27 ; 0,31$; e 0,42 , respectivamente, nos estratos inferior, médio e superior (Quadro 2). O procedimento final de classificação idicou que $100 \%$ das classes de altura total foram corretamente classificadas nos estratos de altura total (Quadro 2).

Na classe I de estoque, as espécies de maior valor de posição sociológica (\%) e ocorrentes simultaneamente nos estratos inferior, médio e superior foram Lecythis idatimon Aubl. (59,2\%), Rinorea guianensis Aubl. (17,5\%) e Eschweilera coriacea Mart. ex Berg. (8,3\%).

Em média, na classe I de estoque, foram observadas 25,4 árvores/ha $(8,4 \%)$ com altura total entre 8,0 e $15,0 \mathrm{~m}$ no estrato inferior (I); 208,6 árvores/ha $(68,7 \%)$ com altura total entre 15,1 e $23,0 \mathrm{~m}$ no estrato médio (II); e 69,6 árvores/ha (22,9\%) com altura total entre 23,1 e $40 \mathrm{~m}$ no estrato superior (III) (Quadro 3). Em geral, maiores valores de densidade absoluta (n/ ha) e de número de espécies (S) foram observados no estrato médio (II). Porém, a diversidade de espécies (H') foi maior no estrato superior (III), que mais contribuiu para elevar o valor do índice de diversidade da comunidade $\left(\mathrm{H}^{\prime}=3,98\right)$.

O estrato superior (III) apresentou uma densidade de 69,6 árvores/ha, com uma área basal de 13,89 m²/ ha e volume de $201,40 \mathrm{~m}^{3} / \mathrm{ha}$ (Quadro 3). Isso pode ser explicado pelo fato de o referido estrato englobar os indivíduos com maiores diâmetros e alturas.

A análise de agrupamento efetuada na classe de estoque II (Figura 2) indicou que as classes de altura total de 1 a 7 formam o estrato inferior (I), as classes de altura total de 8 a 15 o estrato médio (II) e as classes de altura total de 16 a 25 o estrato superior (III). 
Quadro 2 - Número de classes de altura total e porcentagens de classificação correta obtidos da análise discriminante Table 2 - Number of total height strata and percentage of correct classification abtaneid discrinant analysis

\begin{tabular}{lccccc}
\hline Estrato & \multicolumn{2}{c}{ Classificação em Estratos de Altura } & Total & Classificação (\%) \\
\cline { 2 - 5 } & $\begin{array}{c}\text { Inferior (I) } \\
\mathrm{P}=0,27\end{array}$ & $\begin{array}{c}\text { Médio (II) } \\
\mathrm{P}=0,31\end{array}$ & $\begin{array}{c}\text { Superior (III) } \\
\mathrm{P}=0,42\end{array}$ \\
\hline Inferior (I) & 7 & 0 & 0 & 7 & 100 \\
Médio (II) & 0 & 8 & 0 & 8 & 11 \\
Superior (III) & 0 & 0 & 11 & 26 & 100 \\
Total & 7 & 8 & 11 & 100 \\
\hline
\end{tabular}

Quadro 3 - Estimativas da densidade absoluta (n/ha), da dominância absoluta $\left(\mathrm{m}^{2} / \mathrm{ha}\right)$, do volume de fuste ( $\left.\mathrm{m}^{3} / \mathrm{ha}\right)$, do número de espécies $(\mathrm{S})$, da diversidade máxima $\left(\mathrm{H}_{\text {máx }}\right)$ e do índice de Shannon-Weaver $\left(\mathrm{H}^{\prime}\right)$ por estrato de altura total, classe I de estoque. Fazenda Tracajás, município de Paragominas, Estado do Pará

Table 3 - Average estimatites of absolute density $(\mathrm{n} / \mathrm{ha})$, absolute dominance $\left(\mathrm{m}^{2} / \mathrm{ha}\right)$, stems the volume ( $\left.\mathrm{m}^{3} / \mathrm{ha}\right)$, species the number $(S)$, maximal diversity $\left(H_{\max }\right)$ and diversity index of Shannon-Weaver $\left(H^{\prime}\right)$ per total height stratum for Tracajás Farm, municipality Paragominas, Pará state

\begin{tabular}{cccccccc}
\hline Estrato & Amplitude $(\mathrm{m})$ & $\mathrm{n} / \mathrm{ha}$ & $\mathrm{m}^{2} / \mathrm{ha}$ & $\mathrm{m} / \mathrm{ha}$ & $\mathrm{S}$ & $\mathrm{H}_{\text {máx }}$ & $\mathrm{H}^{\prime}$ \\
\hline I & $8,0-15,0$ & 25,4 & 0,75 & 7,23 & 51 & 3,93 & 3,40 \\
II & $15,1-23,0$ & 208,6 & 11,79 & 140,22 & 135 & 4,91 & 3,80 \\
III & $23,1-40,0$ & 69,6 & 13,89 & 201,40 & 90 & 4,50 & 3,99 \\
Total & & 303,6 & 26,43 & 348,85 & 157 & 5,10 & 3,98 \\
\hline
\end{tabular}

A análise discriminante apresentou probabilidades de 0,28 ; 0,32; e 0,40, respectivamente, nos estratos inferior, médio e superior (Quadro 4). O procedimento final de classificação evidenciou que $100 \%$ das classes de altura total foram corretamente classificadas nos estratos de altura total (Quadro 4).

Na classe II de estoque, as espécies de maior valor de posição sociológica (\%) e ocorrentes simultaneamente nos estratos inferior, médio e superior da floresta foram Lecythis idatimon (67,6\%) e Eschweilera coriacea $(14,8 \%)$.

Em média, na classe II de estoque foram observadas
52,8 árvores/ha $(17,7 \%)$ com altura total entre 8,0 e $15,0 \mathrm{~m}$ no estrato inferior (I), 208,4 árvores/ha (69,8\%) com altura total entre 15,1 e $23,0 \mathrm{~m}$ no estrato médio (II) e 37,2 árvores/ha (12,5\%) com altura total entre 23,1 e 36,0m no estrato superior (III) (Quadro 5). Maiores valores de densidade absoluta (n/ha), dominância absoluta ( $\left.\mathrm{m}^{2} / \mathrm{ha}\right)$, volume de fuste comercial $\left(\mathrm{m}^{3} / \mathrm{ha}\right)$ e do número de espécies $(\mathrm{S})$ foram para o estrato médio (II). Porém, maior diversidade (H') foi estimada no estrato III.

A diversidade estimada pelo índice de ShannonWeaver (H') de 3,78 caracterizou alta diversidade de espécies no estrato superior (Quadro 5).

Quadro 4 - Número de classes de altura total e porcentagens de classificação correta obtido da análise discriminante Table 4-Number of total height strata and percentage of correct classification abtaneid discrinant analysis

\begin{tabular}{lccccc}
\hline Estrato & \multicolumn{2}{c}{ Classificação em Estratos de Altura } & Total & Classificação(\%) \\
\cline { 2 - 5 } & $\begin{array}{c}\text { Inferior (I) } \\
\mathrm{P}=0,28\end{array}$ & $\begin{array}{c}\text { Médio (II) } \\
\mathrm{P}=0,32\end{array}$ & $\begin{array}{c}\text { Superior (III) } \\
\mathrm{P}=0,40\end{array}$ & 7 & 100 \\
\hline Inferior (I) & 7 & 0 & 0 & 8 & 100 \\
Médio (II) & 0 & 8 & 0 & 10 & 100 \\
Superior (III) & 0 & 0 & 10 & 25 & 100 \\
Total & 7 & 8 & 10 & 25 \\
\hline
\end{tabular}


Quadro 5 - Estimativas da densidade absoluta (n/ha), da dominância absoluta ( $\left.\mathrm{m}^{2} / \mathrm{ha}\right)$, do volume de fuste ( $\left.\mathrm{m}^{3} / \mathrm{ha}\right)$, do número de espécies $(\mathrm{S})$, da diversidade máxima $\left(\mathrm{H}_{\text {máx }}\right)$ e do índice de Shannon-Weaver $\left(\mathrm{H}^{\prime}\right)$ por estrato de altura total, classe II de estoque. Fazenda Tracajás, município de Paragominas, Estado do Pará

Table 5 - Average estimatites of absolute density ( $\mathrm{n} / \mathrm{ha})$, absolute dominance $\left(\mathrm{m}^{2} / \mathrm{ha}\right)$, stems the volume $\left(\mathrm{m}^{3} / \mathrm{ha}\right)$, species the number $(S)$, maximal diversity $\left(H_{\max }\right)$ and diversity index of Shannon-Weaver $\left(H^{\prime}\right)$ per total height stratum for Tracajás Farm, municipality Paragominas, Pará state

\begin{tabular}{cccccccc}
\hline Estrato & Amplitude $(\mathrm{m})$ & $\mathrm{n} / \mathrm{ha}$ & $\mathrm{m}^{2} / \mathrm{ha}$ & $\mathrm{m}^{3} / \mathrm{ha}$ & $\mathrm{S}$ & $\mathrm{H}_{\text {máx }}$ & $\mathrm{H}^{\prime}$ \\
\hline I & $8,0-15,0$ & 52,8 & 1,69 & 15,42 & 61,0 & 4,11 \\
II & $15,1-23,0$ & 208,4 & 15,97 & 179,04 & 126,0 & 4,84 \\
III & $23,1-36,0$ & 37,2 & 9,11 & 126,18 & 65,0 & 4,17 & 3,68 \\
Total & & 298,4 & 26,77 & 320,64 & 151,0 & 5,00 & 3,78 \\
\hline
\end{tabular}

A análise de agrupamento executada na classe de estoque III (Figura 3 ) apontou que as classes de altura total de 1 a 10 formam o estrato inferior (I), as classes de 11 a 18 o estrato médio (II) e as classes de 19 a 23 o estrato superior (III).

A análise discriminante indicou probabilidades de 0,43 ; 0,35 ; e 0,22 , respectivamente, nos estratos inferior, médio e superior (Quadro 6). O procedimento final de classificação evidenciou que $100 \%$ das classes de altura total foram corretamente classificadas nos estratos de altura total (Quadro 6).

Na classe III de estoque, as espécies com maior valor de posição sociológica (\%) e ocorrentes simultaneamente nos estratos inferior e médio foram Lecythis idatimon (42,4\%), Eschweilera coriacea (20,2\%) e Rinorea guianensis $(17,6 \%)$.

Uma espécie presente no estrato ou dossel superior e que apresenta indivíduos simultaneamente nos estratos médio e inferior tem sustentabilidade ambiental.

$\mathrm{Na}$ classe III de estoque (Quadro 7) foram observadas 159,8 árvores/ha $(53,44 \%)$ com altura total entre 8,0 e 18,0 m no estrato inferior (I), 131,2 árvo- res/ha $(43,9 \%)$ com altura total entre 18,1 e $26,0 \mathrm{~m}$ no estrato médio (II) e 8,0 árvores/ha $(2,7 \%)$ com altura total entre 26,1 e 35,0 m no estrato superior (III). Enfim, na classe III de estoque os estratos inferiores de altura total possuem maiores números de indivíduos.

Os estoques de biomassa expressos em termos de $\mathrm{m}^{2} /$ ha e $\mathrm{m}^{3} /$ ha foram maiores no estrato médio, devido ao número de indivíduos com maiores diâmetros (Quadro 7). Apesar de o estrato inferior (I) apresentar maior número de indivíduos (159,8/ha), os valores de área basal $\left(7,52 \mathrm{~m}^{2} / \mathrm{ha}\right)$ e do volume $\left(45,457 \mathrm{~m}^{3} / \mathrm{ha}\right)$ foram baixos. Isso se deve ao número de indivíduos com menores tamanhos (diâmetros e alturas). A densidade absoluta (n/ha), o número de espécies (S) e o índice diversidade de Shannon-Weaver (H') decresceram do sentido do estrato inferior para o estrato superior. Esse fato pode ser explicado pela distribuição dos indivíduos nos estratos arbóreos e pela capacidade produtiva do local.

As espécies de maior valor de posição sociológica (\%) ocorrentes nas classes I, II e III de estoque foram Lecythis idatimon e Eschweilera coriacea.

Quadro 6 - Número de classes de altura total e porcentagens de classificação correta obtidos da análise discriminante Table 6 - Number of total height strata and percentage of correct classification abtaneid discrinant analysis

\begin{tabular}{|c|c|c|c|c|c|}
\hline \multirow[t]{2}{*}{ Estrato } & \multicolumn{3}{|c|}{ Classificação em Estratos de Altura } & \multirow[b]{2}{*}{ Total } & \multirow[b]{2}{*}{ Classificação(\%) } \\
\hline & $\begin{array}{c}\text { Inferior }(\mathrm{I}) \\
\mathrm{P}=0,43\end{array}$ & $\begin{array}{l}\text { Médio(II) } \\
\mathrm{P}=0,35\end{array}$ & $\begin{array}{c}\text { Superior(III) } \\
\mathrm{P}=0,22\end{array}$ & & \\
\hline$\overline{\text { Inferior (I) }}$ & 10 & 0 & 0 & 10 & 100 \\
\hline Médio (II) & 0 & 8 & 0 & 8 & 100 \\
\hline Superior (III) & 0 & 0 & 5 & 5 & 100 \\
\hline Total & 10 & 8 & 5 & 23 & 100 \\
\hline
\end{tabular}


Quadro 7 - Estimativas da densidade absoluta (n/ha), da dominância absoluta ( $\mathrm{m}^{2} / \mathrm{ha}$ ), do volume de fuste ( $\left.\mathrm{m}^{3} / \mathrm{ha}\right)$, do número de espécies $(\mathrm{S})$, da diversidade máxima $\left(\mathrm{H}_{\text {máx }}\right)$ e do índice de Shannon-Weaver $\left(\mathrm{H}^{\prime}\right)$ por estrato de altura total, classe III de estoque. Fazenda Tracajás, município de Paragominas, Pará

Table 7 -Average estimatites of absolute density $(\mathrm{n} / \mathrm{ha})$, absolute dominance $\left(\mathrm{m}^{2} / \mathrm{ha}\right)$, volume $\left(\mathrm{m}^{3} / \mathrm{ha}\right)$, species the number $(S)$, maximal diversity $\left(H_{\max }\right)$ and diversity index of Shannon-Weaver $\left(H^{\prime}\right)$ per total height stratum for Tracajás Farm, municipality Paragominas, Pará state

\begin{tabular}{cccccccc}
\hline Estrato & Amplitude $(\mathrm{m})$ & $\mathrm{n} / \mathrm{ha}$ & $\mathrm{m}^{2} / \mathrm{ha}$ & $\mathrm{m}^{3} / \mathrm{ha}$ & $\mathrm{S}$ & $\mathrm{H}_{\text {máx }}$ & $\mathrm{H}^{\prime}$ \\
\hline I & $8,0-18,0$ & 159,8 & 7,52 & 45,55 & 118 & 4,77 & 3,75 \\
II & $18,1-26,0$ & 131,2 & 14,74 & 180,33 & 92 & 4,52 & 3,62 \\
III & $26,1-35,0$ & 8,0 & 2,85 & 75,01 & 23 & 3,14 & 3,03 \\
Total & & 299,0 & 25,14 & 300,97 & 139 & 5,00 & 3,82 \\
\hline
\end{tabular}

Os valores de H' de 3,98; 3,80; e 3,82 indicam elevada diversidade de espécies nas classes de estoque I, II e III, respectivamente. Tais valores foram superiores aos encontrados em estudos realizados por Ribeiro et al. (1999), Maciel et al. (2000), Martins Pinto (2000) e Yared et al. (2000), em floresta tropical de terra firme, na Amazônia.

As áreas com maior capacidade produtiva (classe I de estoque) apresentaram maior riqueza e diversidade $\left(H^{\prime}=3,98\right)$. Essa diversidade foi mais elevada no estrato superior (III) de altura total, enquanto a riqueza foi maior no estrato médio (II).

A estratificação vertical das classes de florestas permitiu analisar a composição florística e a estrutura em termos de riqueza, diversidade, densidade, dominância e, sobretudo, de estoques de volumes total e comercial. Isso indica que áreas de florestas naturais com diferente capacidade de estoques de volume potencialmente comercial apresentam estruturas verticais distintas.

\section{CONCLUSÕES}

- As técnicas de análise de agrupamento e discriminante são eficientes para a estratificação vertical de florestas heterogêneas.

- A estratificação das árvores em estratos de altura total permite melhor conhecimento da composição e da estrutura da floresta em termos de riqueza e diversidade de espécies, crescimento e produção de biomassa, revelando características biológicas e ecológicas das espécies florestais.

- As áreas com menor estoque de volume de madeira potencialmente comercial apresentaram maior rique- za e diversidade de espécies no estrato inferior de alturas totais. As áreas com maior estoque de volume de madeira potencialmente comercial exibiram maior riqueza no estrato médio e diversidade de espécies no estrato superior, respectivamente.

\section{REFERÊNCIAS BIBLIOGRÁFICAS}

\author{
BROWER, J. E.; ZAR, J. H. Field \& \\ laboratory methods for general \\ ecology. Dubuque: Wm. C. Brown publishers, \\ 1984. $226 \mathrm{p}$.
}

BURTON, A. J.; RAMM, C. W.; PREGITZER, K. S. Use of multivariate methods in forest research site selection. Canadian Journal of Forest Research, v. 21, p. 1573-1580, 1991.

CALEGÁRIO, N.; SCOLFORO, J. R. S.; SOUZA, A. L. Estratificação em alturas para floresta natural heterogênea: uma proposta metodológica.

Cerne, v. 1, n. 1, p. 58-63, 1994.

HUNTER Jr., M. L. Wildlife forests, and forestry: principles of managing forests for biological diversity. New Jersey: Prentice-Hall, 1990. $370 \mathrm{p}$.

\section{LAMPRECHT, H. Silvicultura nos}

Trópicos: ecossistemas florestais e respectivas espécies arbóreas - possibilidades e métodos de aproveitamento sustentado. Eschborn: Deustsche Gessells chaft für technisch Zusammenarbeti (GTZ) GmbH, 1990. 343 p.

LATHAM, P. A.; ZUURING, H. R.; COBLE, D. W. A method for quantifying vertical forest structure. Forest Ecology and Management, v. 104, p. 157-170, 1998. 
LONGHI, S. J. A estrutura de uma floresta natural de Araucaria angustifolia (Ber.) $O$. Ktze, no sul do Brasil. 1980. 198 f. Dissertação (Mestrado em Ciência Florestal) Universidade Federal do Paraná, Curitiba, 1980.

MACIEL, M. N. M.; QUEIROZ, W. T.; OLIVEIRA, F. A. Parâmetros fitossociológicos de uma floresta tropical de terra firme na Floresta Nacional de Caxiuanã-PA. Revista Ciências Agrárias, n. 34, p. 85-106, 2000.

\section{MARISCAl Flores, E. J. Potencial} produtivo e alternativas de manejo sustentável de um fragmento de mata atlântica secundária, município de Viçosa, Minas Gerais. 1993. $165 \mathrm{f}$. Dissertação (Mestrado em Ciência Florestal) Universidade Federal de Viçosa, Viçosa, 1993.

MARTins PINTO, A. C. M. Análise de danos de colheita de madeira em floresta tropical úmida sob regime de manejo florestal sustentável na Amazônia Ocidental. 2000. 165 f. Dissertação (Mestrado em Ciência Florestal) Universidade Federal de Viçosa, Viçosa, 2000.

MUELLER-DOMBOIS, D.; ELLEMBERG, $\mathrm{H}$. Aims and methods of vegetation ecology. New York: Jonh Wiley \& Sons, 1974. $547 \mathrm{p}$.
QUEIROZ, W. T. Análise de fatores ("Factor Analysis") pelo método da máxima verossimilhança: aplicação ao estudo da estrutura de florestas tropicais. 1984. $109 \mathrm{f}$. Tese (Doutorado em Engenharia Florestal) - Universidade Federal do Paraná, Curitiba, 1984.

RIBEIRO, R. J. et al. Estudo fitossociológico nas regiões de Carajás e Marabá - PA, Brasil. Acta Amazônica, v. 29, n. 2, p. 207-222, 1999.

SANQUETA, C. R. Análise da estrutura vertical de florestas através do diagrama h-M. Ciência Florestal, v. 5, n. 1, p. 55-68, 1995.

SOUZA, D. R. et al. Emprego da análise multivariada para estratificação vertical de florestas ineqüiâneas. Revista Árvore, v. 27, n. 1, p. 59-63, 2003.

VEGA, C. L. Observaciones ecológicas sobre los bosques de roble de la sierra Boyoca, Columbia. Turrialba, v. 16, n. 3, p. 286-296, 1966.

YARED, J. A. G.; COUTO, L.; LEITE, H. G. Diversidade de espécies em florestas secundária e primária, sob efeito de diferentes sistemas silviculturais, na Amazônia Oriental. Revista Árvore, v. 24, n. 1, p. 83-90, 2000. 